\title{
MicroRNA Expression Aberration Associated with Bronchopulmonary Dysplasia in Preterm Infants: A Preliminary Study
}

\author{
Yen-Tzu Wu PhD, Wei J Chen ScD, Wu-Shiun Hsieh MD, Po-Nien Tsao PhD, \\ Sung-Liang Yu PhD, Chi-Yu Lai MS, Wen-Chung Lee PhD, and Suh-Fang Jeng ScD PT
}

BACKGROUND: Because environmental insults and genetic factors account for the variance in the risk of bronchopulmonary dysplasia (BPD) in very low birth weight (VLBW, birth weight $<1,500 \mathrm{~g}$ ) preterm infants, the search for BPD biomarkers has begun to focus on the regulators of non-coding RNA such as microRNAs (miRNAs). Therefore, this study aimed to identify potential miRNAs involved in the pathogenesis of BPD in VLBW preterm infants. METHODS: A case-control study (15 subjects with BPD and 15 sex-matched control subjects without BPD) was conducted to investigate the expression profiles of 365 miRNAs in the peripheral blood of VLBW preterm infants at 36 weeks post-menstrual age (called the older-age set). The expression levels of identified miRNAs were further evaluated in a subsample of blood collected during the first 2 weeks post-natal age (called the younger-age set). Possible biological functions and pathways implicated in the target genes regulated by the miRNAs were explored using database predictions. RESULTS: A 4-miRNA signature (miR-152, miR-30a-3p, miR-133b, and miR-7) with aberrant expression levels at 36 weeks, derived from a supervised classification with internal cross-validation, discriminated the subjects with BPD from those without BPD with an accuracy of 0.91 . The discriminative accuracy of the 4 miRNAs was supported by random permutations of either the disease status or the number of miRNAs selected (both $P<.001$ ). A down-regulation change of $m i R-152$ and $m i R-30 a-3 p$ expression levels and an up-regulation change of $m i R-133 b$ and $m i R-7$ expression levels were found in the older-age set, compared to the younger-age set. CONCLUSIONS: This is the first study to identify blood-based miRNAs associated with BPD. The findings provide information regarding the roles of these biomarkers in the development of BPD in VLBW preterm infants. Key words: bronchopulmonary dysplasia; microRNA; peripheral blood; biomarkers; expression profiling. [Respir Care 2013; 58(9):1527-1535. (C) 2013 Daedalus Enterprises]

\section{Introduction}

Bronchopulmonary dysplasia (BPD) is an important chronic lung disease in preterm infants with very low birth weight (VLBW, birth weight $<1,500 \mathrm{~g}$ ). ${ }^{1,2}$ Recent advances in the prevention and management of this respiratory illness, such as antenatal steroid treatment, exogenous surfactant therapy, and gentle ventilator strategies, have

\footnotetext{
Dr Wu is affiliated with the School and Graduate Institute of Physical Therapy; Dr Hsieh and Dr Tsao are affiliated with the Department of Pediatrics; Dr Yu is affiliated with the Department of Clinical Laboratory Sciences and Medical Biotechnology; and Dr Jeng is affiliated with the School and Graduate Institute of Physical Therapy, National Taiwan University Hospital College of Medicine, Taipei, Taiwan. Dr Chen is affiliated with the Graduate Institute of Epidemiology and Preventive Medicine; Dr Lai is affiliated with the Graduate Institute of Epidemiology and Preventive Medicine; and Dr Lee is affiliated with the Graduate Institute of Epidemiology and Preventive Medicine, National Taiwan University College of Public Health, Taipei, Taiwan.
}

This study was supported by National Taiwan University Hospital (study NTUH-98-S-1085), National Taiwan University (study 97HP0071), and the Taiwan National Health Research Institute (studies NHRI-EX989519PI and NHRI-EX101-10106PI). The authors have disclosed no conflicts of interest.

Correspondence: Suh-Fang Jeng ScD PT, School and Graduate Institute of Physical Therapy, National Taiwan University College of Medicine, 17 Xu-Zhou Road, Taipei 100, Taiwan. E-mail: jeng@ntu.edu.tw.

DOI: $10.4187 /$ respcare.02166 
changed the underlying pathology of BPD from severe structural disruption to developmental arrest of the neonatal lung. ${ }^{3-5}$ The new form of BPD frequently occurs in extremely preterm infants with pathological features, including impaired alveolarization, vascular growth abnormalities, and mild inflammation. 5,6

In addition to environmental insults (eg, hyperoxia exposure and respiratory therapies) to the immature lung in preterm infants, genetic factors may also account for a portion of the variation in BPD. ${ }^{7,8}$ Although several association studies have examined the roles of genetic variants in inflammatory regulation and surfactant synthesis in BPD, the results have failed to show consistent associations. ${ }^{9-17}$ In developmentally appropriate BPD animal models, altered expression levels of certain transcription factors for protein-coding RNA have been linked to alveolarization, angiogenesis, or remodeling of the lung extracellular matrix. ${ }^{18-22}$ However, BPD pathogenesis in human infants remains incompletely understood.

Instead of targeting DNA or protein-coding RNA in $\mathrm{BPD}$, recent advances in molecular genetics have provided a new strategy for understanding the post-transcriptional regulation of gene expression via non-coding RNAs, in particular microRNAs (miRNAs), which have a regulatory effect on the translation of messenger RNA and protein production. ${ }^{23}$ To date, approximately 1,600 human miRNAs have been identified and sequenced. ${ }^{24}$ Approximately $30 \%$ of messenger RNAs are targeted by miRNAs, and aberrant expression of miRNAs can lead to many disease states. ${ }^{25,26}$ Although recent animal studies have identified several miRNAs in the developmental process of the embryonic lung, and alterations in miRNA expression profiles are associated with BPD, ${ }^{27-31}$ information regarding the role of miRNAs in BPD in human infants is limited.

This study, therefore, aimed to investigate miRNA expression levels in the peripheral blood of VLBW preterm infants with BPD and sex-matched controls at 36 weeks post-menstrual age (called the older-age set). The expression levels of the differentially expressed miRNAs was further examined in a subsample of blood collected during the first 2 weeks post-natal age (called the younger-age set) to elucidate the evolution of miRNA expression during BPD development. The possible functions and biological mechanisms implicated by the target genes regulated by these miRNAs were also explored using database prediction.

\section{Methods}

This study was approved by the ethics committees of the participating hospitals. Written informed consent was obtained from the father or mother after a complete description of the study.

\section{QUICK LOOK}

\section{Current knowledge}

Bronchopulmonary dysplasia (BPD) is a chronic lung disease in preterm infants with very low birth weight. Recent advances in prevention and management of BPD have changed the underlying pathology to a syndrome including impaired alveolarization, vascular growth abnormalities, and mild inflammation.

\section{What this paper contributes to our knowledge}

Down-regulation of 2 microRNAs (miR-152 and $m i R-30 a-3 p)$, and up-regulation of 2 others ( $m i R-133 b$ and $m i R-7)$, were found in peripheral blood samples from very low birth weight infants. Those 4 miRNAs and the related target genes provide insight for future investigation of the pathogenesis of BPD.

\section{Subjects}

From September 2008 to December 2009, we prospectively enrolled VLBW preterm infants who were admitted to the neonatal ICU at the National Taiwan University Hospital and Mackey Memorial Hospital in Taipei, Taiwan. The recruitment was conducted during the first postnatal week. The inclusion criteria included gestational age $<33$ weeks, birth weight $<1,500 \mathrm{~g}$, absence of congenital and chromosomal abnormalities, and born in or admitted to the neonatal ICU within 7 days of birth. Patients were excluded if their parents were of non-Taiwanese ethnicity, maternal age was $<18$ years, or there was a maternal history of substance abuse or psychiatric disorder. BPD was graded for its severity at 36 weeks post-menstrual age, according to the consensus definition of the United States National Institutes of Health. ${ }^{3}$

Of the 54 eligible VLBW preterm infants, 53 (98\%) participated, and 1 (2\%) refused to participate in this study. After enrollment, 50 infants survived to 36 weeks postmenstrual age; 20 had BPD and 30 did not. The incidence rate of BPD in our infants (37\%) was comparable to that reported by the participating hospitals $(20-40 \%)$. We collected $500 \mu \mathrm{l}$ of arterial blood twice from each subject, in conjunction with routine daily blood tests. The first blood draw was scheduled during the first 2 weeks of post-natal age (called the younger-age set), and the second blood draw was conducted at 36 weeks post-menstrual age (called the older-age set).

The perinatal and demographic data of the VLBW preterm infants were obtained from the infants' medical charts or via parental interview during hospitalization. The perinatal data included birth variables, respiratory variables, 
and neonatal morbidities. The birth variables included sex, gestational age, birth weight, and birth set. The respiratory variables included the duration of ventilation and CPAP use. The neonatal morbidities included intraventricular hemorrhage, periventricular leukomalacia, patent ductus arteriosus, retinopathy of prematurity, necrotizing enterocolitis, and sepsis. The demographic data included sex and maternal education and occupation.

\section{RNA Extraction and MicroRNA Quantification}

Approximately 2-4 $\mu \mathrm{g}$ of RNA from mononuclear leukocyte was extracted from each blood sample (RiboPure blood kit, Applied Biosystems/Life Technologies, Carlsbad, California). The RNAs of the older-age set were assayed for the expression of 365 human miRNAs (Multiplex RT and TaqMan low density array human microRNA panel v1.0, and 7900 real-time RT-PCR system, Applied Biosystems/Life Technologies, Carlsbad, California), using RNU48 as an endogenous control. The RNAs of the younger-age set were quantified using the qRT-PCR for the expression levels of the individual miRNAs identified from the older-age set.

The expression level of each miRNA from the arraybased profiling was quantified through the normalized threshold cycle number $\left(\Delta C_{t}\right)$, in which

$$
\Delta \mathrm{C}_{\mathrm{t}}=\left[\mathrm{C}_{\mathrm{t}}(\text { miRNA) }]-\left[\mathrm{C}_{\mathrm{t}}(\mathrm{U} 48)\right]\right.
$$

and the relative expression level was calculated as $2^{-\left(\Delta C_{t}\right)}$, which is commonly used in genome-wide miRNA profiling studies. ${ }^{32}$ The fold-change in the expression of miRNA between 2 groups was calculated as $2^{-\left(\Delta \Delta \mathrm{C}_{t}\right)}$.

\section{Statistical Analyses}

For group comparisons of perinatal and demographic variables between the VLBW preterm infants with versus without BPD, the Fisher exact test was used for categorical variables, and the Wilcoxon signed rank test was used for continuous variables. Significant variables were considered as potential confounders for subsequent analyses of the relationship of miRNA expression levels to BPD occurrence.

To select differentially expressed miRNAs from the older-age set, the Wilcoxon rank-sum test was used to compare the continuous data of expression levels, and the Fisher exact test to compare the binary data of detectability between groups, based on a liberal threshold $P$ of 0.2 . The markers identified from univariate analyses were then subjected to the stepwise logistic regression analysis to select a cluster of miRNAs that could discriminate the subjects with BPD from those without BPD. Furthermore, the leave- one-out cross-validation and 2 types of permutations (permutation of disease status and number of markers selected) were conducted to evaluate the robustness of discrimination by the identified miRNA signature. ${ }^{33}$ Subsequently, the fold-change in the relative expression of miRNA and the direction of fold-change from the younger-age set to the older-age set were examined in the subsample with RNAs available at both time points.

The potential target genes regulated by the miRNAs were assessed using the miRWALK algorithm. ${ }^{34}$ The software Ingenuity Pathways Analysis (IPA, Ingenuity Systems, Redwood City, California), which contains global molecular network data, was used to explore the biological and functional pathway for the target genes. All statistical analyses were performed with statistics software (SAS 9.1, SAS Institute, Cary, North Carolina), and a $P$ value of $<.05$ was considered significant.

\section{Results}

\section{Sample Characteristics}

Of the 50 subjects, the first blood draw was successfully conducted in 21: 11 subjects with BPD (10 with moderate BPD and 1 with severe BPD) and 10 control subjects without BPD, within the first 2 post-natal weeks to form the younger-age set (Table 1). The second blood draw was successfully performed in 44 subjects at 36 weeks postmenstrual age. Fifteen subjects with BPD (14 with moderate BPD and 1 with severe BPD) and 15 sex-matched control subjects without BPD were selected to form the older-age set. In both sets, the subjects with BPD had younger gestational ages and lower birth weights and required longer ventilation and CPAP use than did the subjects without BPD (all $P<.05$ ). Furthermore, the subjects with BPD were more likely to have patent ductus arteriosus (both $P<.05$ ). Analysis of the respiratory data between the subjects with BPD and a subgroup of subjects without $\operatorname{BPD}(n=10)$ with comparable gestational ages $(<31$ weeks) indicated a longer use of ventilation and CPAP in the former than in the latter (all $P<.05$, data not shown).

\section{miRNAs Identified From the Older-Age Set}

For the older-age set, the blood samples tended to be collected earlier for the subjects with BPD than for the subjects without BPD (post-natal age $37 \pm 11 \mathrm{~d}$ vs $47 \pm 12 \mathrm{~d}$ ), although the difference did not reach significance $(P=.09)$. Of the 365 miRNAs examined in the older-age set, $210(58 \%)$ were detected in the infants, and $155(42 \%)$ were undetectable. The heat maps of 365 miRNAs in terms of the proportion of infants in each 
Table 1. Perinatal and Demographic Characteristics of Very Low Birth Weight Preterm Infants With and Without Bronchopulmonary Dysplasia

\begin{tabular}{|c|c|c|c|c|}
\hline \multirow{2}{*}{ Characteristics } & \multicolumn{2}{|c|}{ Older-Age Set } & \multicolumn{2}{|c|}{ Younger-Age Set } \\
\hline & $\begin{array}{c}\text { BPD } \\
n=15\end{array}$ & $\begin{array}{c}\text { No BPD } \\
n=15\end{array}$ & $\begin{array}{c}\text { BPD } \\
n=11\end{array}$ & $\begin{array}{c}\text { No BPD } \\
n=10\end{array}$ \\
\hline Gestational age, median (range) weeks & $28(24-30)^{*}$ & $31(27-32)^{*}$ & $28(24-30) \dagger$ & $31(27-32) \dagger$ \\
\hline Birth weight, median (range) $\mathrm{g}$ & $1,060(506-1,440)^{*}$ & $1,314(1,040-1,478)^{*}$ & $1,020(628-1,340) \dagger$ & $1,296(1,000-1,478) \dagger$ \\
\hline Twin or multiple births, no. (\%) & $5(33)$ & $6(40)$ & $5(45)$ & $6(60)$ \\
\hline Duration of ventilation, median (range) $d$ & $7(0-71)^{*}$ & $0(0-3)^{*}$ & $7(0-71) \dagger$ & $0(0-2) \dagger$ \\
\hline Duration of CPAP, median (range) d & $34(0-58)^{*}$ & $4(0-11)^{*}$ & $32(0-56) \dagger$ & $4(0-11) \dagger$ \\
\hline \multicolumn{5}{|l|}{ Intraventricular hemorrhage, no. (\%) } \\
\hline Normal & $10(67)$ & $9(60)$ & $8(72)$ & $6(60)$ \\
\hline Grade I-II & $4(26)$ & $6(40)$ & $1(9)$ & $4(40)$ \\
\hline Grade III-IV & $1(7)$ & $0(0)$ & $1(9)$ & $0(0)$ \\
\hline Periventricular leukomalacia, no. (\%) & $5(33)$ & $2(13)$ & $1(9)$ & $1(10)$ \\
\hline Patent ductus arteriosus, no. (\%) & $11(73)^{*}$ & $1(7)^{*}$ & $7(64) \dagger$ & $1(10) \dagger$ \\
\hline Necrotizing enterocolitis, no. (\%) & $1(7)$ & $0(0)$ & $0(0)$ & $0(0)$ \\
\hline Sepsis, no. (\%) & $1(7)$ & $2(13)$ & $1(9)$ & $1(10)$ \\
\hline Male, no. (\%) & $6(40)$ & $6(40)$ & $4(36)$ & $5(50)$ \\
\hline Maternal education, median (range) y & $14(12-16)$ & $16(12-18)$ & $16(12-18)$ & $16(12-18)$ \\
\hline \multicolumn{5}{|l|}{ Maternal occupation, no. (\%) } \\
\hline Housewife or unskilled labor & $8(53)$ & $7(47)$ & $2(18)$ & $7(70)$ \\
\hline Technician & $4(27)$ & $6(40)$ & $5(45)$ & $3(30)$ \\
\hline Professional & $3(20)$ & $2(13)$ & $4(36)$ & $0(0)$ \\
\hline $\begin{array}{l}\text { *P<.05 in comparing the } 2 \text { groups in this set. } \\
\dagger P<.05 \text { in comparing the } 2 \text { groups in this set. } \\
\text { BPD }=\text { bronchopulmonary dysplasia }\end{array}$ & & & & \\
\hline
\end{tabular}

group with detectable expression are illustrated in Figure 1, using the Generalized Association Plots program. ${ }^{35}$

For 210 detectable miRNAs, the expression levels did not differ between the subjects with versus without BPD. When miRNA expression was dichotomized as detectable or not, differential detectability between groups was found in 4 miRNAs. The stepwise logistic regression analysis revealed that $m i R-133 b$ and $m i R-7$ had significantly higher detectability in the subjects with BPD than in those without, whereas $m i R-152$ and $m i R-30 a-3 p$ had significantly lower detectability in the subjects with BPD (all $P<.05$, Table 2).

The utility of these 4 miRNAs in discriminating the subjects with BPD from those without was evaluated using multivariable logistic regression analysis, with a leaveone-out cross-validation procedure. The 4-miRNA signature was associated with the greatest accuracy value, with an area under the curve of the receiver operating characteristic of 0.91 (Fig. 2). The sensitivity and specificity for the optimal combination of the 4 miRNAs (expression of $m i R-133 b$ and $m i R-7$ together with non-expression of $m i R-152$ and $m i R-30 a-3 p$ ) were 0.87 and 0.80 , respectively. Because there were high correlations among potential confounding perinatal variables, only some were included as covariates in the analysis. After adjustment for the effect of birth weight and patent ductus arteriosus, the specificity slightly increased, to 0.87 , and the sensitivity remained unchanged.

The robustness of BPD discrimination by the 4-miRNA signature was further evaluated using permutations, in 2 ways. First, when the disease status of the subjects was permuted 100,000 times, none of the areas under the curve derived from the permuted samples (mean $\pm \mathrm{SD}$ $0.63 \pm 0.07)$ exceeded the observed value of 0.91 $(P<.001)$. Second, when a certain number of miRNAs (range 3-9) were randomly selected, the average of areas under the curve (range $0.44-0.73$ ) was found to be smaller than those observed for the 4-miRNA signature.

\section{Expression of 4 MicroRNAs in the Younger-Age and Older-Age Sets}

For the 16 infants (7 subjects with BPD and 9 subjects without BPD) whose RNA expression levels were available in both the younger-age and older-age sets, the fold change in the expression levels of the 4 miRNAs were compared (Fig. 3). For the younger-age set, the fold change in the expression levels between the BPD and the nonBPD group was positive for $m i R-152$ and negative for $m i R-30 a-3 p, m i R-133 b$, and $m i R-7$. For the older-age set, the fold change in the expression levels was negative for $m i R-152$ and $m i R-30 a-3 p$ and positive for $m i R-133 b$ and 


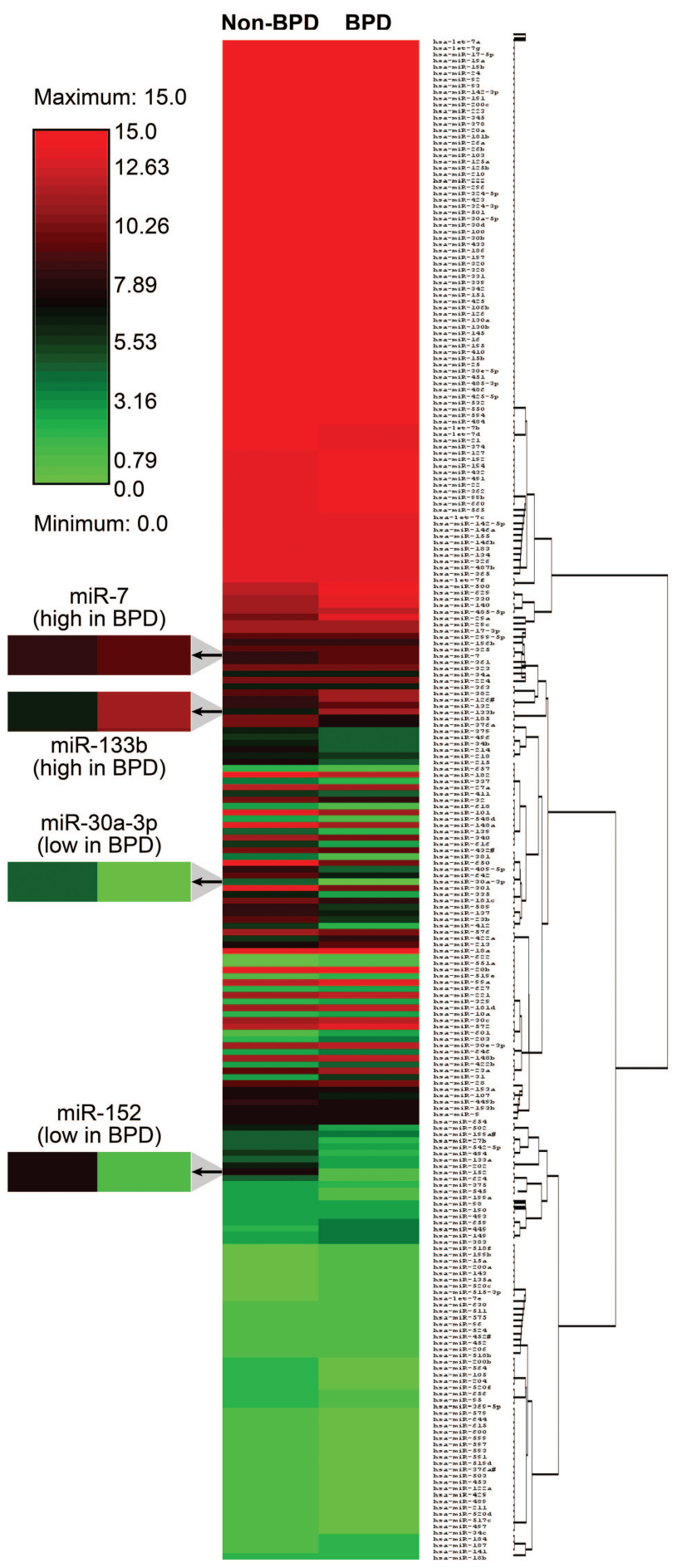

Fig. 1. MicroRNA (miRNA) expression profiling of 365 miRNAs in very low birth weight preterm infants of the older-age set, with and without bronchopulmonary dysplasia (BPD). The heat maps of individual miRNAs for each group are presented as the proportion of infants with detectable miRNA expression, with higher proportions (more red in color) indicating more infants showing expression. Two miRNAs (miR-7 and miR-133b) showed higher expression levels (more red in color), and the others (miR-152 and miR-30a$3 p$ ) showed lower expression levels (more green in color) in the BPD group. $m i R-7$. Analysis of the fold change from the younger-age set to the older-age set revealed a down-regulation in the expression of $m i R-152$ (from 1.2 to -4.8 ) and $m i R-30 a-3 p$ (from -2.1 to -2.7 ) and an up-regulation in the expression of $m i R-133 b$ (from -4.9 to 1.2 ) and $m i R-7$ (from -2.3 to 1.6$)$.

\section{Target Genes and Functional Relevance of the 4-miRNA Signature}

Of the 4 miRNAs, $m i R-152$, $m i R-30 a-3 p$, and $m i R-7$ are included in the human database miRNAMap with detectable expression in lung tissue. ${ }^{36}$ The miRWALK algorithm indicated that 7,239 target genes were relevant to the 4 miRNAs, with 66 being shared target genes. The functional pathway analysis showed that the shared target genes may be related to organ and embryonic development as well as developmental disorders (all $P<.001$, Table 3 ). Among them, GLI3 and NFIB were the most significant target genes involved in lung development (both $P<.001$ ). Canonical biological pathway analysis revealed that the pathways with the most significant changes were the Wnt/ $\beta$-catenin and axon guidance signaling pathways (both $P=.04)$.

\section{Discussion}

To our knowledge, this is the first study to investigate the peripheral blood of VLBW preterm infants in early post-natal life for differentially expressed miRNAs that have an association with BPD. We identified 4 miRNAs with expression levels at 36 weeks post-menstrual age that differed between VLBW preterm infants with and without BPD. Two time-point comparisons of the expression levels of these miRNAs, at 36 weeks post-menstrual age with those at the first 2 weeks post-natal age, further revealed a down-regulation change in $m i R-152$ and $m i R-30 a-3 p$ expression, together with an up-regulation change in $m i R$ $133 \mathrm{~b}$ and $m i R-7$ expression during the early stage of BPD. Our results provide a preliminary understanding of the roles of miRNAs in the development of BPD in VLBW preterm infants.

The 4-miRNA signature had acceptable discriminative value for the detection of BPD in VLBW preterm infants. Our observation concerning the higher discriminative accuracy of the combined altered expressions of these 4 miRNAs than that of a single miRNA was consistent with the findings of a previous study. ${ }^{33}$ Because miRNAs are regulators closer in temporal sequence to outcome than is genetic information encoded in the genome, the aberrantly expressed miRNAs may be useful biomarkers for BPD. Among the 4 differentially expressed miRNAs, $m i R-30 a-3 p$ and $m i R$-30 were highly up-regulated in mouse lung during the period of lung septation. ${ }^{27,31}$ In humans, miR-30a- 
Table 2. The 4 miRNAs With Aberrant Expressions at 36 Weeks Post-Menstrual Age*

\begin{tabular}{|c|c|c|c|c|c|}
\hline \multirow[t]{2}{*}{ miRNA } & \multirow[t]{2}{*}{ Chromosomal Region } & \multirow[t]{2}{*}{ Location } & \multicolumn{2}{|c|}{$\begin{array}{c}\text { Detectability of miRNA } \\
\text { Expression } \\
\text { no. }(\%)\end{array}$} & \multirow[t]{2}{*}{$P \dagger$} \\
\hline & & & $\begin{array}{c}\text { BPD } \\
n=15\end{array}$ & $\begin{array}{c}\text { No BPD } \\
n=15\end{array}$ & \\
\hline$m i R-152$ & $17 \mathrm{q} 21.32$ & Intron & $1(7)$ & $8(53)$ & .005 \\
\hline$m i R-30 a-3 p$ & $6 q 13$ & Intron & $0(0)$ & $5(33)$ & .03 \\
\hline$m i R-133 b$ & $6 \mathrm{p} 12.2$ & Intron & $12(80)$ & $7(47)$ & .04 \\
\hline$m i R-7$ & $9 q 21.32$ & Intron & $10(67)$ & $5(33)$ & .04 \\
\hline
\end{tabular}

* Derived from a stepwise logistic regression analysis in a sample of 15 very low birth weight preterm infants with bronchopulmonary dysplasia (BPD) and 15 sex-matched very low birth weight preterm infants without BPD. The data are from the human database of miRNAMAP (http://mirnamap.mbc.nctu.edu.tw).

$\dagger$ Based on stepwise logistic regression analysis.

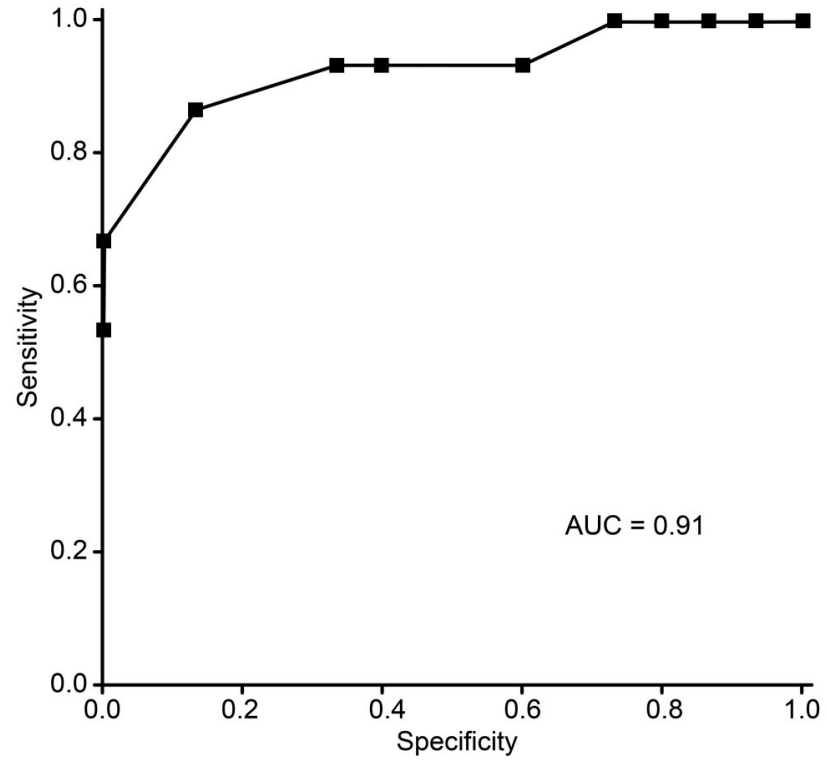

Fig. 2. Receiver operating characteristic curve for the discrimination of bronchopulmonary dysplasia by the 4-microRNA signature model.

$3 p, m i R-133 b$, and $m i R-7$ are associated with adult lung diseases, with $m i R-30 a-3 p$ and $m i R-133 b$ being downregulated and $m i R-7$ being up-regulated in lung tumor tissue of adults with adenocarcinoma. ${ }^{37-39}$ Furthermore, $m i R$ 152 is associated with childhood asthma. ${ }^{40}$ Thus, these 4 miRNAs appear to be associated with a variety of lung diseases.

BPD in preterm infants has been postulated to result from lung injuries related to prolonged use of ventilation and oxygen therapy in the neonatal period. ${ }^{3,5,6}$ In this study preterm infants with BPD had longer ventilation duration (median $7 \mathrm{~d}$ vs $0 \mathrm{~d}$ ) and CPAP use (median $34 \mathrm{~d}$ vs $4 \mathrm{~d}$ ) than those without BPD. Furthermore, the 4 miRNAs showed different expression levels in the peripheral blood of preterm infants with BPD at 36 weeks post-menstrual age when respiratory support was in use. A study in hu-

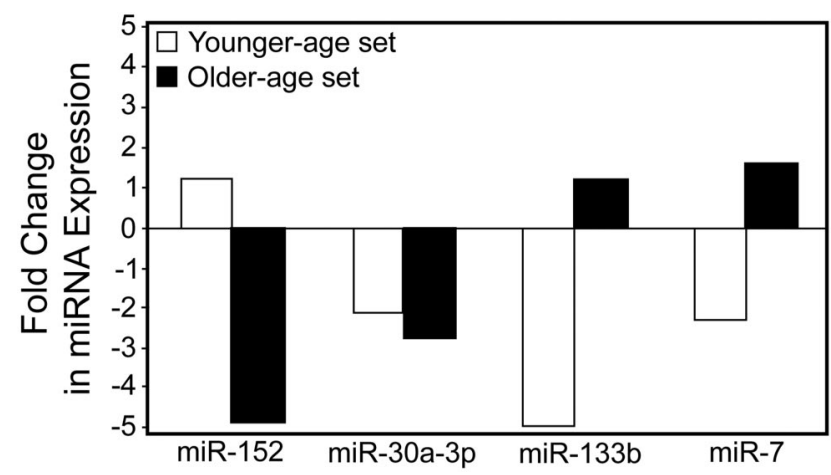

Fig. 3. Expression pattern of the 4-microRNA (miRNA) signature in the younger-age and older-age sets in a subsample of 16 infants (7 subjects with BPD and 9 subjects without BPD) with their RNA expression levels examined at both time points. miR-152 and miR$30 a-3 p$ were down-regulated, and miR-133b and miR-7 were upregulated in the BPD group, compared to the non-BPD group in the older-age set, compared to the younger-age set.

mans found that hypoxic events altered the expression of several miRNAs (eg, miR-152) in colonic cells. ${ }^{41}$ An animal study screening for lung miRNA profiles in rats after chronic hypoxia showed aberrant expression of several miRNA markers, including $m i R-30 . .^{42}$ It is possible that the aberrant expression of miRNAs might relate to hypoxic or hyperoxic insults from ventilator and oxygen use.

It is noteworthy that our preliminary results suggest a developmental shift in the expression pattern of these 4 miRNAs (ie, down-regulation of $m i R-152$ and $m i R-30 a-3 p$ and up-regulation of $m i R-133 \mathrm{~b}$ and $m i R-7)$ during the neonatal period. A genome-wide profiling study in mice found 21 differently expressed miRNAs in hyperoxia-exposed BPD mice. Both $m i R-30$ and $m i R-133$ showed a more than 2-fold increase in BPD mice, compared to control mice, and the expression increased with advancing age, at 2, 7 , and 21 post-natal days. ${ }^{31} \mathrm{An}$ increase of oxygen exposure with the ages might be one possible factor relating to the change in miRNA expression. Our finding concerning 
Table 3. Biological Functions and Diseases Related to the Target Genes in the 4-miRNA Signature

\begin{tabular}{lll}
\hline \hline \multicolumn{1}{c}{ Category } & \multicolumn{1}{c}{ Function and Disease* } & \multicolumn{1}{c}{ Involved Target Gene $\dagger$} \\
\hline Organ development & Organ development & APTA7A, BCL11B, GLI3, NFIB, SLC1A2 \\
& Lung development & GLI3, NFIB \\
& Forebrain development & GLI3, NFIB \\
& Brain development & GLI3, NFIB, SLC1A2 \\
Embryonic development & Neural tube development & ENAH, GLI3 \\
& Embryonic tissue development & ENAH, GLI3 \\
& Cell death of embryonic cell lines & AAK1, MTF1 \\
Developmental disorder & Neurological process of embryonic tissue & ENAH, GLI3 \\
& Developmental disorder & ATP7A, GLI3, NFIB, SLC1A2, VANGL1 \\
* The listed functions and diseases were determined via Ingenuity Pathways Analysis. & \\
$\dagger$ The target genes of the 4-miRNA signature involved in the biological function or disease. & \\
All $P<.001$ & & \\
\hline
\end{tabular}

$m i R-30 a-3 p$ down-regulation in preterm infants with BPD was in contrast to the up-regulation observed in the mouse BPD model. ${ }^{31}$ Future investigation of the evolution of miRNA expression at various time points after oxygen use may elucidate how oxygen therapy might alter the expression levels of the 4 miRNAs in preterm infants with BPD.

In addition to the lung injury caused by oxidative stress, developmental disorders of alveolarization and dysmorphic pulmonary vasculature have recently been recognized as pathology hallmarks of the new form of BPD. ${ }^{6}$ While the lung tissue is not readily accessible for investigation of miRNAs in the surviving preterm infants, blood-based expression profiling is increasingly being undertaken as a noninvasive method to investigate BPD biomarkers. It is plausible that these BPD-related expression aberrations of miRNAs in the peripheral mononuclear leukocyte might relate to the pathologic changes of fewer alveolar capillary and dysmorphic pulmonary vasculature in the preterm infants with BPD. The insult to the gas-exchange function may exert its influence on the gene expression of peripheral lymphocytes via cytokines, growth factors, or transmembrane molecules. ${ }^{43,44}$

An intriguing finding from the functional pathway analyses among database-derived target genes for the 4 miRNAs indicated that the 2 most important target genes, $N F I B$ and GLI3, are involved in lung development, such as alveolarization and pulmonary vasculature, consistent with the pathological hallmarks of the new form of BPD. ${ }^{6,45}$ For example, the transcription factors encoded by NFIB genes were highly expressed in embryonic mouse lung and might be involved in fetal lung maturation, ${ }^{46,47}$ and $G L I 3$, a transcription factor of the sonic hedgehog cascade, is critical for the patterning of early lung morphogenesis in mice. ${ }^{48,49}$ Moreover, NFIB-deficient mice die shortly after birth, and their severe lung hypoplasia resembles BPD. ${ }^{47,50}$ In future work the miRNA-target genes must be validated using either exogenous (eg, over-expression of certain
miRNAs in lung cell lines to examine the protein expression level of the target genes) or endogenous (eg, manipulation of miRNAs under physiological conditions) miRNA experiments. ${ }^{51}$

The Wnt/ $\beta$-catenin signaling pathway identified from the canonical biological pathway analysis among the miRNA-targeted genes can regulate airway epithelial differentiation and vascular smooth muscle proliferation in the developing lung. ${ }^{52}$ Disruption of the $\mathrm{Wnt} / \beta$-catenin signaling pathway in primordial mouse lung results in a failure to form lung buds and thereby arrests proliferation in the vascular smooth muscle of the lungs. ${ }^{53,54}$ Further investigation should examine how the target genes and the 4-miRNA signature modulate the $\mathrm{Wnt} / \beta$-catenin signaling pathway in an animal or cell line model of BPD.

The results of this study should be interpreted with some limitations in mind. First, because the number of human miRNAs $(n=365)$ examined in this study was much less than what is currently available (more than 1,600 to date), further assessment of the miRNAs not included in our experiment is warranted. Second, despite the acceptable discriminative accuracy of the blood-based miRNAs for $\mathrm{BPD}$, these biomarkers might not represent the entire spectrum of regulatory changes in BPD pathophysiology. Direct examination of the lung tissue is needed to clarify the underlying mechanisms. Third, VLBW preterm infants without BPD were treated as the controls in this study. Nevertheless, the individual differences among the subjects with BPD and those without BPD in terms of the growth and nutrition status, neonatal care, and other medical treatments during the neonatal period were not accounted for in this study. Experimental factors such as the miRNA assay conditions may further influence the miRNA expression levels and, hence, the fold-change in relative expression of miRNA when using the data of controls as the reference. Finally, the sample size in this study was 
relatively small; therefore, future validation of our results in a larger sample is necessary.

\section{Conclusions}

In summary, this study demonstrates the potential utility of a mononuclear leukocyte-based miRNA signature as a biomarker for BPD in VLBW preterm infants. As the pathology features of the new form of BPD have been changed to a developmental disorder of the neonatal lung, the identified miRNAs and related target genes in this study provide insight for future investigation of the pathogenesis of BPD in VLBW preterm infants.

\section{ACKNOWLEDGMENTS}

We thank the infants and their parents for participating in the study and the medical and nursing staff of the Neonatal ICU at National Taiwan University Hospital and Mackey Memorial Hospital for their assistance in patient recruitment and data collection.

\section{REFERENCES}

1. Walsh MC, Szefler S, Davis J, Allen M, Van Marter L, Abman S, et al. Summary proceedings from the bronchopulmonary dysplasia group. Pediatrics 2006;117(3 Pt 2):S52-S56.

2. Fanaroff AA, Stoll BJ, Wright LL, Carlo WA, Ehrenkranz RA, Stark AR, et al. Trends in neonatal morbidity and mortality for very low birthweight infants. Am J Obstet Gynecol 2007;196(2):147.e141-e148.

3. Jobe AH, Bancalari E. Bronchopulmonary dysplasia. Am J Respir Crit Care Med 2001;163(7):1723-1729.

4. Kinsella JP, Greenough A, Abman SH. Bronchopulmonary dysplasia. Lancet 2006;367(9520):1421-1431.

5. Baraldi E, Filippone M. Chronic lung disease after premature birth. N Engl J Med 2007;357(19):1946-1955.

6. Bhandari A, Bhandari V. Pitfalls, problems, and progress in bronchopulmonary dysplasia. Pediatrics 2009;123(6):1562-1573.

7. Bhandari V, Bizzarro MJ, Shetty A, Zhong X, Page GP, Zhang H, et al. Familial and genetic susceptibility to major neonatal morbidities in preterm twins. Pediatrics 2006;117(6):1901-1906.

8. Lavoie PM, Pham C, Jang KL. Heritability of bronchopulmonary dysplasia, defined according to the consensus statement of the national institutes of health. Pediatrics 2008;122(3):479-485.

9. Kazzi SN, Kim UO, Quasney MW, Buhimschi I. Polymorphism of tumor necrosis factor-alpha and risk and severity of bronchopulmonary dysplasia among very low birth weight infants. Pediatrics 2004; 114(2):e243-e248.

10. Yanamandra K, Boggs P, Loggins J, Baier RJ. Interleukin-10-1082 G/A polymorphism and risk of death or bronchopulmonary dysplasia in ventilated very low birth weight infants. Pediatr Pulmonol 2005; 39(5):426-432.

11. Balogh A, Treszl A, Vannay A, Vasarhelyi B. A prevalent functional polymorphism of insulin-like growth factor system is not associated with perinatal complications in preterm infants. Pediatrics 2006; 117(2):591-592.

12. Lin HC, Su BH, Hsu CM, Chou IC, Wu KH, Lin HC, et al. No association between TAP1 DpnII polymorphism and bronchopulmonary dysplasia. Acta Paediatr Taiwan 2005;46(6):341-345.

13. Adcock K, Hedberg C, Loggins J, Kruger TE, Baier RJ. The TNF-alpha -308, MCP-1-2518 and TGF-beta $1+915$ polymorphisms are not associated with the development of chronic lung disease in very low birth weight infants. Genes Immun 2003;4(6):420-426.
14. Strassberg SS, Cristea IA, Qian D, Parton LA. Single nucleotide polymorphisms of tumor necrosis factor-alpha and the susceptibility to bronchopulmonary dysplasia. Pediatr Pulmonol 2007;42(1):29-36.

15. Derzbach L, Bokodi G, Treszl A, Vásárhelyi B, Nobilis A, Rigó JJ. Selectin polymorphisms and perinatal morbidity in low-birthweight infants. Acta Paediatr 2006;95(10):1213-1217.

16. Weber B, Borkhardt A, Stoll-Becker S, Reiss I, Gortner L. Polymorphisms of surfactant protein A genes and the risk of bronchopulmonary dysplasia in preterm infants. Turk J Pediatr 2000;42(3): 181-185.

17. Pavlovic J, Papagaroufalis C, Xanthou M, Liu W, Fan R, Thomas $\mathrm{NJ}$, et al. Genetic variants of surfactant proteins A, B, C, and D, in bronchopulmonary dysplasia. Dis Markers 2006;22(5-6):277-291.

18. Bry K, Whitsett JA, Lappalainen U. IL-1beta disrupts postnatal lung morphogenesis in the mouse. Am J Respir Cell Mol Biol 2007;36(1): $32-42$.

19. Altiok O, Yasumatsu R, Bingol-Karakoc G, Riese RJ, Stahlman MT, Dwyer W, et al. Imbalance between cysteine proteases and inhibitors in a baboon model of bronchopulmonary dysplasia. Am J Respir Crit Care Med 2006;173(3):318-326.

20. Bland RD, Xu L, Ertsey R, Rabinovitch M, Albertine KH, Wynn $\mathrm{KA}$, et al. Dysregulation of pulmonary elastin synthesis and assembly in preterm lambs with chronic lung disease. Am J Physiol Lung Cell Mol Physiol 2007;292(6):L1370-L1384.

21. Tambunting F, Beharry KD, Hartleroad J, Waltzman J, Stavitsky Y, Modanlou HD. Increased lung matrix metalloproteinase-9 levels in extremely premature baboons with bronchopulmonary dysplasia. Pediatr Pulmonol 2005;39(1):5-14.

22. Maniscalco WM, Watkins RH, Pryhuber GS, Bhatt A, Shea C, Huyck H. Angiogenic factors and alveolar vasculature: development and alterations by injury in very premature baboons. Am J Physiol Lung Cell Mol Physiol 2002;282(4):L811-L823.

23. Zhang C. MicroRNomics: a newly emerging approach for disease biology. Physiol Genomics 2008;33(2):139-147.

24. Homo sapiens miRNAs (1872 sequences). miRBase: the microRNA database. http://www.mirbase.org/cgi-bin/mirna_summary.pl?org= hsa. Accessed June 26, 2013.

25. Bentwich I. Prediction and validation of microRNAs and their targets. FEBS Lett 2005;579(26):5904-5910.

26. Friedman JM, Jones PA. MicroRNAs: critical mediators of differentiation, development and disease. Swiss Med Wkly 2009;139 (33-34):466-472.

27. Williams AE, Moschos SA, Perry MM, Barnes PJ, Lindsay MA. Maternally imprinted microRNAs are differentially expressed during mouse and human lung development. Dev Dyn 2007;236(2):572-580.

28. Bhaskaran M, Wang Y, Zhang H, Weng T, Baviskar P, Guo Y, et al. MicroRNA-127 modulates fetal lung development. Physiol Genomics 2009;37(3):268-278.

29. Wang Y, Weng T, Gou D, Chen Z, Chintagari NR, Liu L. Identification of rat lung-specific microRNAs by micoRNA microarray: valuable discoveries for the facilitation of lung research. BMC Genomics 2007;8:29.

30. Lu Y, Okubo T, Rawlins E, Hogan BL. Epithelial progenitor cells of the embryonic lung and the role of microRNAs in their proliferation. Proc Am Thorac Soc 2008;15(3):300-304.

31. Zhang X, Peng W, Zhang S, Wang C, He X, Zhang Z, et al. MicroRNA expression profile in hyperoxia-exposed newborn mice during the development of bronchopulmonary dysplasia. Respir Care 2011; 56(7):1009-1015.

32. Schmittgen TD, Lee EJ, Jiang J, Sarkar A, Yang L, Elton TS, et al. Real-time PCR quantification of precursor and mature microRNA. Methods 2008;44(1):31-38. 


\section{MicroRnA Expression Aberration Associated with Bronchopulmonary DysPlasia}

33. Lai CY, Yu SL, Hsieh MH, Chen CH, Chen HY, Wen CC, et al. MicroRNA expression aberration as potential peripheral blood biomarkers for schizophrenia. PLoS One 2011;6(6):e21635.

34. Dweep H, Sticht C, Pandey P, Gretz N. miRWalk-Database: prediction of possible miRNA binding sites by "walking" the genes of 3 genomes. J Biomed Inform 2011;44(5):839-847.

35. Wu H, M,. A graphical environment for matrix visualization and cluster analysis. Comput Stat Data Anal 2010;54(3):767-778.

36. Hsu SD, Chu CH, Tsou AP, Chen SJ, Chen HC, Hsu PW, et al. miRNAMap 2.0: genomic maps of microRNAs in metazoan genomes. Nucleic Acids Res 2008;36(Database issue):D165-D169.

37. Gao W, Shen H, Liu L, Xu J, Shu Y. MiR-21 overexpression in human primary squamous cell lung carcinoma is associated with poor patient prognosis. J Cancer Res Clin Oncol 2010;137(4):557-566.

38. Crawford M, Batte K, Yu L, Wu X, Nuovo GJ, Marsh CB, et al. MicroRNA 133B targets pro-survival molecules MCL-1 and BCL2L2 in lung cancer. Biochem Biophys Res Commun 2009;388(3):483-489.

39. Navon R, Wang H, Steinfeld I, Tsalenko A, Ben-Dor A, Yakhini Z. Novel rank-based statistical methods reveal microRNAs with differential expression in multiple cancer types. PLoS One 2009;4(11): e8003.

40. Tan Z, Randall G, Fan J, Camoretti-Mercado B, Brockman-Schneider $\mathrm{R}$, Pan L, et al. Allele-specific targeting of microRNAs to HLA-G and risk of asthma. Am J Hum Genet 2007;81(4):829-834.

41. Guimbellot JS, Erickson SW, Mehta T, Wen H, Page GP, Sorscher EJ, et al. Correlation of microRNA levels during hypoxia with predicted target mRNAs through genome-wide microarray analysis. BMC Med Genomics 2009;25:2-15.

42. Caruso P, MacLean MR, Khanin R, McClure J, Soon E, Southgate $\mathrm{M}$, et al. Dynamic changes in lung microRNA profiles during the development of pulmonary hypertension due to chronic hypoxia and monocrotaline. Arterioscler Thromb Vasc Biol 2010;30(4):716-723.

43. Bhatt AJ, Pryhuber GS, Huyck H, Watkins RH, Metlay LA, Maniscalco WM. Disrupted pulmonary vasculature and decreased vascular endothelial growth factor, Flt-1, and TIE-2 in human infants dying with bronchopulmonary dysplasia Am J Respir Crit Care Med 2001; 164(10 Pt 1):1971-1980.

44. Coalson JJ. Pathology of bronchopulmonary dysplasia. Semin Perinatol 2006;30(4):179-184.

45. Thébaud B, Abman SH. Bronchopulmonary dysplasia: where have all the vessels gone? Roles of angiogenic growth factors in chronic lung disease. Am J Respir Crit Care Med 2007;175(10):978-985.

46. Comai G, Boutet A, Neirijnck Y, Schedl A. Expression patterns of the Wtx/Amer gene family during mouse embryonic development. Dev Dyn 2010;239(6):1867-1878.

47. Grunder A, Ebel TT, Mallo M, Schwarzkopf G, Shimizu T, Sippel AE, et al. Nuclear factor I-B (Nfib) deficient mice have severe lung hypoplasia. Mech Dev 2002;112(1-2):69-77.

48. Li Y, Zhang H, Choi SC, Litingtung Y, Chiang C. Sonic hedgehog signaling regulates Gli3 processing, mesenchymal proliferation, and differentiation during mouse lung organogenesis. Dev Biol 2004; 270(1):214-231.

49. Zhang M, Wang H, Teng H, Shi J, Zhang Y. Expression of SHH signaling pathway components in the developing human lung. Histochem Cell Biol 2010;134(4):327-335.

50. Steele-Perkins G, Plachez C, Butz KG, Yang G, Bachurski CJ, Kinsman SL, et al. The transcription factor gene Nfib is essential for both lung maturation and brain development. Mol Cell Biol 2005; 25(2):685-698

51. Xiao F, Zuo Z, Cai G, Kang S, Gao X, Li T. miRecords: an integrated resource for microRNA-target interactions. Nucleic Acids Res 2009;37(Database issue):D105-D110.

52. Pongracz JE, Stockley RA. Wnt signalling in lung development and diseases. Respir Res 2006;26:7-15.

53. Cohen ED, Ihida-Stansbury K, Lu MM, Panettieri RA, Jones PL, Morrisey EE. Wnt signaling regulates smooth muscle precursor development in the mouse lung via a tenascin C/PDGFR pathway. J Clin Invest 2009;119(9):2538-2549.

54. Chen F, Cao Y, Qian J, Shao F, Niederreither K, Cardoso WV. A retinoic acid-dependent network in the foregut controls formation of the mouse lung primordium. J Clin Invest 2010;120(6):2040-2048. 